\title{
Unidirectional Acoustic Sensor Based on the Particle Velocity Gradient
}

\author{
Shiduo Yu ${ }^{1,2}$, Graciano Carrillo Pousa ${ }^{2}$, Daniel Fernández Comesaña ${ }^{2}$ and Yixin Yang ${ }^{1}$ \\ ${ }^{1}$ School of Marine Science and Technology, Northwestern Polytechnical University, \\ No.127 Youyixi Road, Xi'an, China, \\ chitianysd@foxmail.com, carrillo@microflown.com,fernandez@microflown.com, yxyang@nwpu.edu.cn \\ ${ }_{2}^{2}$ Microflown Technologies, 6824 BV Arnhem, the Netherlands
}

\begin{abstract}
Particle velocity sensors have a directivity pattern of "figure-of-eight", whose sensitivity is proportional to the cosine of the sound angle of arrival. Similar to the sound pressure gradient probe, a first order particle velocity gradient probe could be created by using two closely spaced particle velocity sensors. The combination of the properties of both particle velocity sensors and a first order particle velocity gradient can yield to a unidirectional particle velocity sensor which can play a key role in many cases where the rejection of the acoustic excitation coming from undesired directions is critical. The aim of this work is to summarize the theory of unidirectional particle velocity sensors both in frequency domain and time domain, and demonstrate it experimentally with two closely spaced particle velocity sensors.
\end{abstract}

Key words: unidirectional sensor, particle velocity, vector sensor and particle velocity gradient

\section{Introduction}

Acoustic sensors with good directional characteristics are key tools for applications such as speech intelligibility and source localization where the presence of background noise strongly influences the measurement system performance. Therefore, it is rather imperative to develop an acoustic sensor which can reject the acoustic excitation coming from undesired directions. In this case, the unidirectional sensor is then an ideal solution.

Since 1932, extensive research was undertaken on cardioid unidirectional microphones based on the first order sound pressure gradient principle [1]. In the next few decades, the first order unidirectional microphones became more and more complex. Meanwhile, researchers started to investigate second and higher order unidirectional micro-phones in order to enhance directional characteristics.

With particle velocity sensors emerging, the development of a unidirectional sensor based on particle velocity gradient became feasible. After the Microflown sensor was introduced (see Fig.1) [2], its reduced dimensions and the high similarities between sensor elements enable the creation of a unidirectional sensor based on particle velocity gradient.

The directivity of sound pressure sensors (i.e. microphones) is generally omnidirectional. In contrast, particle velocity sensors (also called
Microflown sensors) have a frequency independent figure-of-eight directivity. Fig. 2 illustrates a comparison of the Microflown sensor directivity pattern and a conventional pressure microphone. As shown, Microflown sensors follow a cosine shape and its phase changes sign each 180 degrees.

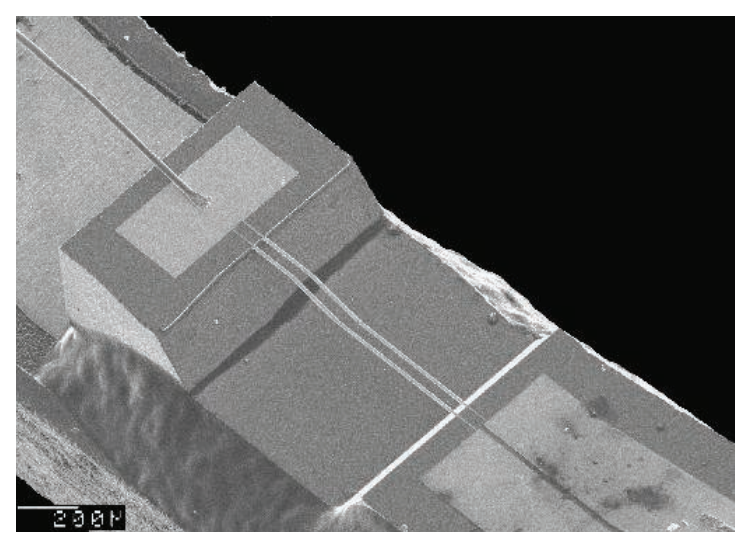

Fig. 1. Picture of a Microflown sensor. The reference line at the bottom-left corner of the figure corresponds to $200 \mu \mathrm{m}(0.2 \mathrm{~mm})$.

As proposed in [3], a unidirectional velocity sensor can be created by combining a particle velocity sensor with a particle velocity gradient sensor. The principle followed is similar to the traditional cardioid microphone which is composed by a sound pressure microphone and a sound pressure gradient microphone. In the following sections a review of the basic 
theory is given along with some experimental results which prove the feasibility of unidirectional sensors based on particle velocity gradient.

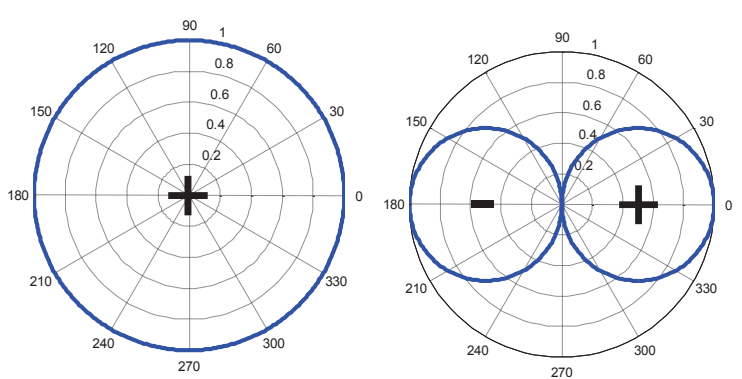

Fig. 2. Directivity pattern of a classical omnidirectional microphone (left); and directivity pattern of the Microflown sensor (right).

\section{Theory of the First Order Particle Velocity Gradient Sensor}

Similar to the sketch shown in Fig. 3, particle velocity $\mathbf{U}$ can be decomposed into two orthogonal components $\mathbf{U}_{x}$ and $\mathbf{U}_{y}$. In the following derivation $U, U_{x}$, and $U_{y}$ denote the module of $\mathbf{U}, \mathbf{U}_{x}$ and $\underline{\mathbf{U}}_{y}$, respectively.

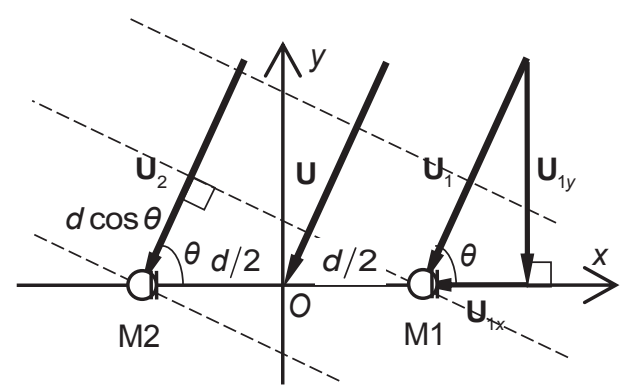

Fig. 3. Plane wave impinging on the unidirectional sensor combined by Microflown sensors M1 and M2. The signal $U_{1 x}$ received by $M 1$ is the module of $x$ component $\mathbf{U}_{1 x}$

In other words, if there is a particle velocity sensor at origin $O, U_{x}$ represents the signal received by a one-dimensional sensor at that particular point. The $x$-component $U_{x}$ can be calculated by multiplying the particle velocity module $U$ and the directivity pattern $b(\theta)$ which, in this case, equals $\cos \theta$. Consequently, the particle velocity signal detected by the sensors are

$$
U_{1 x}(\omega)=U_{1}(\omega) b(\theta)=U_{1}(\omega) \cos \theta
$$

and

$$
U_{2 x}(\omega)=U_{2}(\omega) \mathrm{b}(\theta)=U_{2}(\omega) \cos \theta,
$$

where $\omega$ is the angular frequency, $\theta$ is the angle of incidence. The Directional Index (DI) of each particle velocity sensor can be define as

$$
\begin{aligned}
D I & =10 \log _{10} \frac{\int_{0}^{\pi} \sin \theta \mathrm{d} \theta}{\int_{0}^{\pi} b^{2}(\theta) \sin \theta \mathrm{d} \theta} \\
& =10 \log _{10} \frac{\int_{0}^{\pi} \sin \theta \mathrm{d} \theta}{\int_{0}^{\pi} \cos ^{2} \theta \sin \theta \mathrm{d} \theta}
\end{aligned}
$$

$\approx 4.77 \mathrm{~dB}$.

When the distance $d$ between sensors M1 and M2 is sufficiently short, the acoustic signal coming from any direction can be considered as plain wave, which means these two sensors perceive a very similar signal with a small delay. Therefore, the phase difference between the particle velocity measured at $\mathrm{M} 1$ and origin $O$ equals $d \cos \theta$. Similarly, the particle velocity at origin $O$ has the same delay with respect to $M 2$, which could be expressed as

$$
U_{1}(\omega)=U(\omega) \exp (\mathrm{i} k / 2 \cdot d \cos \theta)
$$

and

$$
U_{2}(\omega)=U(\omega) \exp (-i k / 2 \cdot d \cos \theta),
$$

where $k$ is the wave number, $d$ is distance between two sensors.

A first order particle velocity gradient can be then computed using a finite difference approximation. Therefore, the output of first order particle velocity gradient sensor is

$$
\frac{\mathrm{d} U_{x}(\omega)}{\mathrm{d} x}=\frac{U_{1 x}(\omega)-U_{2 x}(\omega)}{d}
$$

When $|k d / 2|<<1$, the sum and difference can be obtained by using Eq. (1), (2), (4), and (5) as

$$
U_{1 x}(\omega)+U_{2 x}(\omega) \approx 2 \cdot U(\omega) \cos \theta
$$

and

$$
\begin{aligned}
U_{1 x}(\omega)-U_{2 x}(\omega) & \approx i k d \cdot U(\omega) \cos ^{2} \theta \\
& =i(\omega / c) d \cdot U(\omega) \cos ^{2} \theta .
\end{aligned}
$$

Then, averaged particle velocity by Eq. (7) and the normalized first order particle velocity gradient by combining Eq. (6) and (8) are

$$
\frac{U_{1 x}(\omega)+U_{2 x}(\omega)}{2} \approx U(\omega) \cos \theta
$$

and

$$
\frac{c}{\mathrm{i} \omega} \cdot \frac{\mathrm{d} U_{x}(\omega)}{\mathrm{d} x}=U(\omega) \cos ^{2} \theta
$$

which means the directivity pattern $b(\theta)$ of first order particle velocity gradient is $\cos ^{2} \theta$. The Directional Index (DI) of first order particle velocity gradient sensor is 


$$
D I=10 \log _{10} \frac{\int_{0}^{\pi} \sin \theta \mathrm{d} \theta}{\int_{\pi}^{0}\left(\cos ^{2} \theta\right)^{2} \sin \theta \mathrm{d} \theta}
$$

$$
\approx 6.99 \mathrm{~dB} \text {. }
$$

\section{Theory of the Unidirectional Sensor based on Particle Velocity Gradient}

One approach for achieving a unidirectional particle velocity sensor (Fig. 5 right) is to combine a one dimensional particle velocity sensor (Fig. 2 right) with its corresponding particle velocity gradient response (Fig. 5 left). The analogous process using a sound pressure approach would require to add the omnidirectional directivity pattern (Fig. 2 left) to the first order sound pressure gradient directivity pattern.
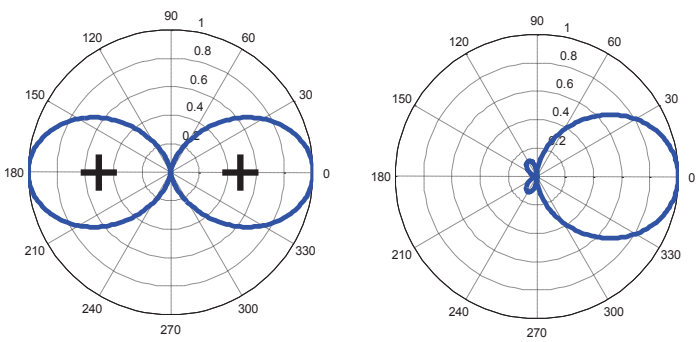

Fig. 4. Directivity pattern of the first order particle velocity gradient sensor (left); Directivity pattern of the unidirectional sensor based on particle velocity gradient (right).

The unidirectional particle velocity expression in frequency domain can be then achieved by relating the previous Eq. (9) and (10), expressed as

$$
\begin{aligned}
& U_{u}(\omega)=U(\omega) \cos ^{2} \theta+U(\omega) \cos \theta \\
& =\frac{c}{\mathrm{i} \omega} \cdot \frac{U_{1 x}(\omega)-U_{2 x}(\omega)}{d}+\frac{1}{2} \cdot\left(U_{1 x}(\omega)+U_{2 x}(\omega)\right) .
\end{aligned}
$$

Then, the unidirectional particle velocity expression in time domain can be obtained as

$$
\begin{aligned}
& u_{u}(t)=u(t) \cos ^{2} \theta+u(t) \cos \theta \\
& =c \cdot \int \frac{u_{1 x}(t)-u_{2 x}(t)}{d} \mathrm{~d} t+\frac{1}{2} \cdot\left(u_{1 x}(t)+u_{2 x}(t)\right),
\end{aligned}
$$

where the variable $t$ denotes time. In Eq. (12) and (13), $\quad\left(U_{1 x}(\omega)-U_{2 x}(\omega)\right) / d \quad$ and $\left(u_{1 x}(t)-u_{2 x}(t)\right) / d$ are particle velocity gradients providing a sufficiently short distance $d$. Therefore, the normalized directivity pattern $b(\theta)$ becomes $\left(\cos \theta+\cos ^{2} \theta\right) / 2$. The Directional Index (DI) of unidirectional sensor based on particle velocity gradient is

$$
D I=10 \log _{10} \frac{\int_{0}^{\pi} \sin \theta d \theta}{\int_{\pi}^{0}\left(\frac{\cos \theta+\cos ^{2} \theta}{2}\right)^{2} \sin \theta d \theta}
$$$$
\approx 8.75 \mathrm{~dB} \text {. }
$$

The difference of Directional Indices between various sensors is described in Tab. 1. As shown, the unidirectional sensor based on particle velocity gradient is superior to the other acoustic sensors reviewed.

Tab. 1: Comparison of directivity Indices.

\begin{tabular}{|c|c|c|}
\hline Category & $\begin{array}{c}\text { Normalized } \\
\text { directivity } \\
\text { pattern b }(\theta)\end{array}$ & $\begin{array}{c}\text { Directional } \\
\text { Index }\end{array}$ \\
\hline Microphone & 1 & $0 \mathrm{~dB}$ \\
\hline $\begin{array}{c}\text { First order sound } \\
\text { pressure sensor }\end{array}$ & $\cos \theta$ & $4.77 \mathrm{~dB}$ \\
\hline $\begin{array}{c}\text { Particle velocity } \\
\text { sensor }\end{array}$ & $\cos \theta$ & $4.77 \mathrm{~dB}$ \\
\hline $\begin{array}{c}\text { First order particle } \\
\text { velocity gradient } \\
\text { sensor }\end{array}$ & $\cos ^{2} \theta$ & $6.99 \mathrm{~dB}$ \\
\hline $\begin{array}{c}\text { Unidirectional } \\
\text { sensor based on } \\
\text { sound pressure } \\
\text { gradient (cardioid) }\end{array}$ & $\frac{1+\cos \theta}{2}$ & $4.77 \mathrm{~dB}$ \\
\hline $\begin{array}{c}\text { Unidirectional } \\
\text { sensor based on } \\
\text { particle velocity } \\
\text { gradient }\end{array}$ & $\frac{\cos \theta+\cos ^{2} \theta}{2}$ & $8.75 \mathrm{~dB}$ \\
\hline
\end{tabular}

\section{Simulations}

The above theory of unidirectional sensor was derived assuming plane waves. This implies that the distance between sensors $d$ should much shorter than the wavelength of sound so that the plane wave approximation still applies. Consequently, it is vital to understand the connection between the distance $d$ and the performance of the unidirectional sensor. Furthermore, a practical implementation of the theory presented above requires to take into account additional factors such as the Signal Noise Ratio (SNR), and the distance between unidirectional sensor and sound source.

Some simulations were performed using the setup sketched in Fig. 5 , where $D$ is the distance between unidirectional sensor and sound source; $d$ is distance between two Microflown sensors and $\theta$ is the sound incident angle. The unidirectional sensor can be created 
by combining the signals of the two onedimensional Microflown sensors M1 and M2.

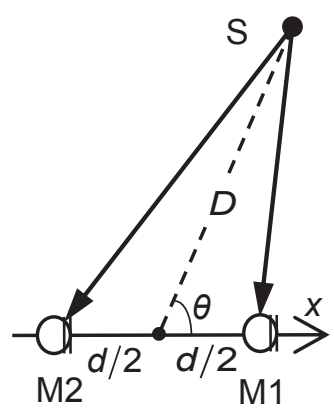

Fig. 5. Sketch of simulation and measurement setup.

All simulation were performed considering standard atmospheric pressure (1.01325 kPa) at temperature of $22{ }^{\circ} \mathrm{C}$. In this case, sound speed is $344.63 \mathrm{~m} / \mathrm{s}$ and air density is 1.1945 $\mathrm{kg} / \mathrm{m}^{3}$. The directional characteristics of the unidirectional sensor mainly depends upon four parameters: frequency, distance between unidirectional sensor and sound source $(D)$, distance between sensors $(d)$, and signal-tonoise ratio (SNR).

Firstly, Fig. 6 presents a comparison of the Directivity Index achieved with several configurations. On the left hand side, the directivity index is evaluated for different frequencies. Furthermore, the performance achieved with several signal-to-noise ratios are displayed on the right hand side of Fig. 6. As shown, when the sensors are separated by 10 $\mathrm{mm}$, the DI curves (c) and (d) are very close to the theoretical limit of $8.75 \mathrm{~dB}$ from $60 \mathrm{~Hz}$ to 8 $\mathrm{kHz}$. However, DI curves (a) and (b) below 100 $\mathrm{Hz}$ decrease to $4.77 \mathrm{~dB}$, which is equal to $\mathrm{DI}$ of particle velocity gradient sensor due to low SNR. On the right hand side of Fig. 6, the DI curves (a), (b), and (c) reach the maximum Directional

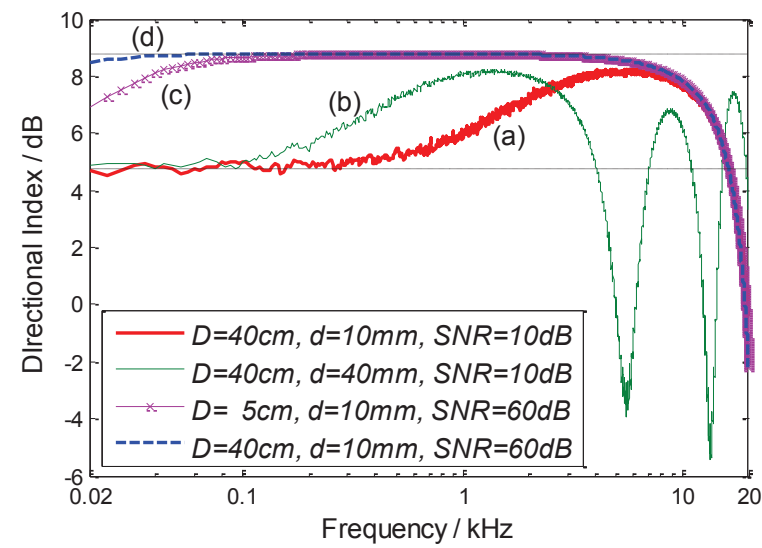

Index, while (d) and (e) are beyond optimum working frequency range for $d=40 \mathrm{~mm}$.

Three main conclusions can be drawn from the simulation results presented in Fig. 6:

- When the distance between particle velocity sensors is shortened, the working frequency range is shifted to higher frequencies (see Fig. 6 left (a) and (b)).

- The distance between the unidirectional sensor and the sound source should be sufficiently far to minimize the influence of the near field of particle velocity that affects the lower frequencies (see Fig. 6 left (c) and (d)).

- A high SNR ensures a good performance of the unidirectional sensor, which is more apparent in the low frequency range (see Fig. 6 right).

In summary, in order to design an effective response in high frequencies, the distance between the particle velocity elements should be sufficiently short. In contrast, a good performance at low frequencies requires a larger separation between the sensor elements. A similar trade-off is found in sound intensity $p$ $p$ probes when selecting microphone spacing.

\section{Experiments}

Many directivity tests were undertaken in order to prove the theory presented above. Fig. 7 shows the Microflown particle velocity sensors used during the tests. The unidirectional system composed by two particle velocity sensors was rotated by 10 degrees, while a loudspeaker was fixed at $40 \mathrm{~cm}$ away from the center of unidirectional system in a non-anechoic testing room. The Signal Noise Ratio (SNR) achieved with the prototype system was nearly of $30 \mathrm{~dB}$.

Fig. 6. Directional Index (DI) curves of unidirectional sensor for different parameters configurations: frequency, distance between unidirectional sensor and sound source (D), distance between sensors (d), and Signal Noise Ratio (SNR). Parameters of right figure are $D=40 \mathrm{~cm}, d=10 \mathrm{~mm}$. 


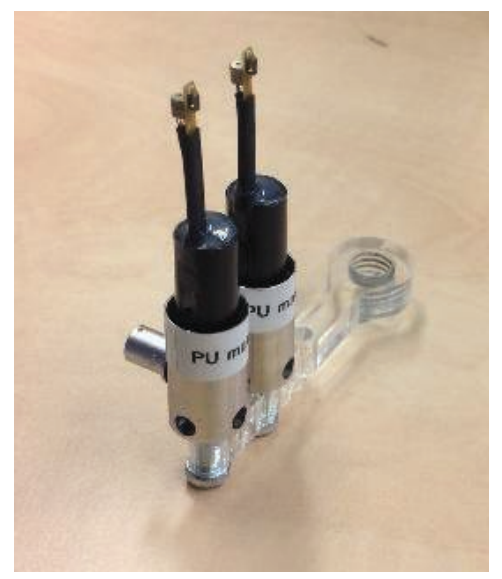

Fig. 7. Picture of the prototype unidirectional velocity sensor composed by two particle velocity elements.

The experimental results are shown in Fig. 8 (b) and (d) for two different sensor separations: 11 $\mathrm{mm}$ and $27 \mathrm{~mm}$. On the other hand, Fig. 8 (a) and (c) present the simulations conducted with the same parameters but in free-field conditions.

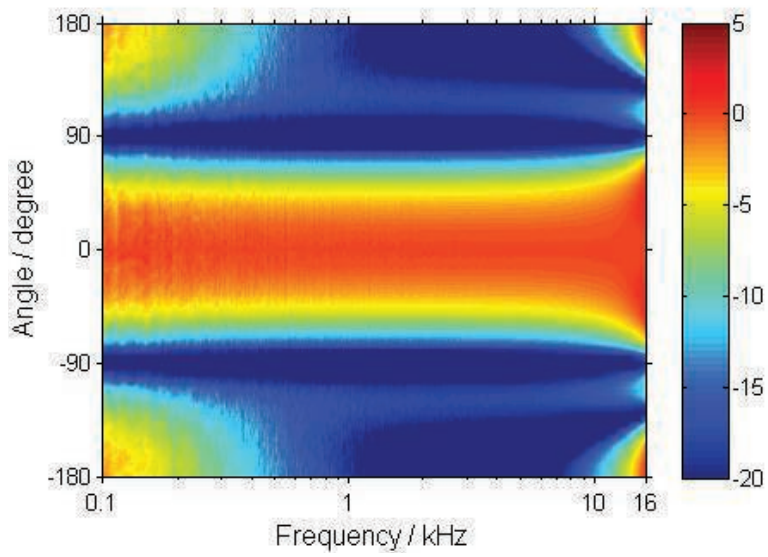

(a) Ideal directivity map in $D=40 \mathrm{~cm}, d=11 \mathrm{~mm}, S N R=30 \mathrm{~dB}$

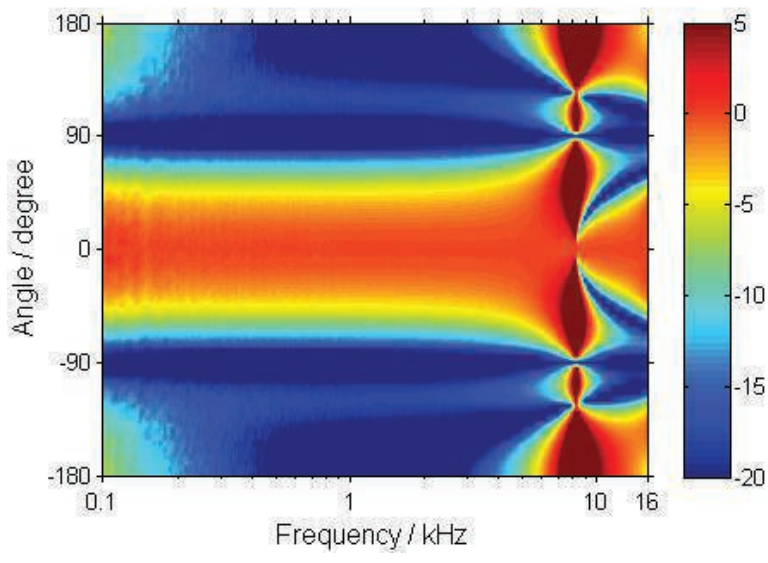

(c) Ideal directivity map in

$D=40 \mathrm{~cm}, d=27 \mathrm{~mm}, S N R=30 \mathrm{~dB}$
As can be seen, in the range from $1 \mathrm{kHz}$ to 16 $\mathrm{kHz}$, the measured and simulated results of unidirectional sensor are very similar. The performance differs in low frequency (below 1 $\mathrm{kHz}$ ). The cause of this difference could be due to the influence of the non-anechoic testing conditions. The impact of reverberation shall be researched in future works.

For frequencies higher than $10 \mathrm{kHz}$ the results are not very stable but follow the same trend as the simulations. One of the reasons that could explain this phenomena could be because of the particle velocity sensor elements were calibrated only below $10 \mathrm{kHz}$.

The results presented above prove not only theoretically but also experimentally the fundamentals of unidirectional sensors based on particle velocity gradient. Further research should be undertaken to extend the frequency range and find optimum configurations for both low and high frequencies.

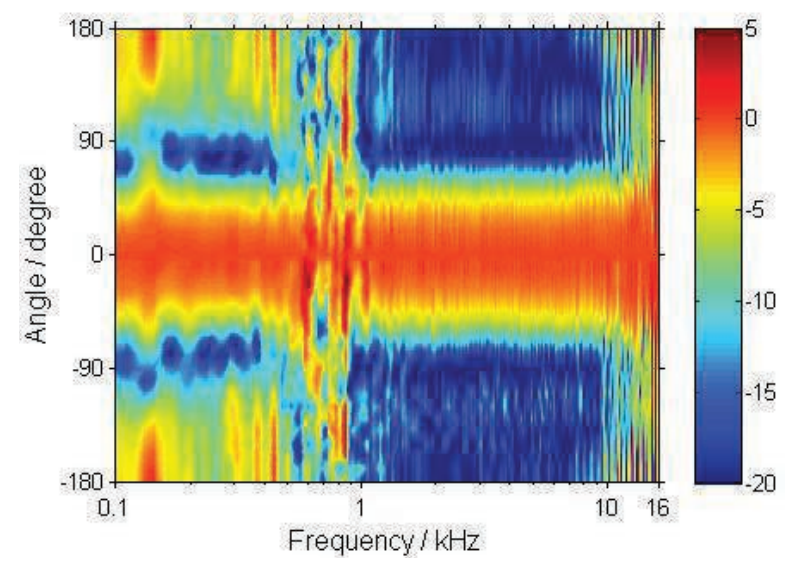

(b) Measured directivity map in $D=40 \mathrm{~cm}, d=11 \mathrm{~mm}$

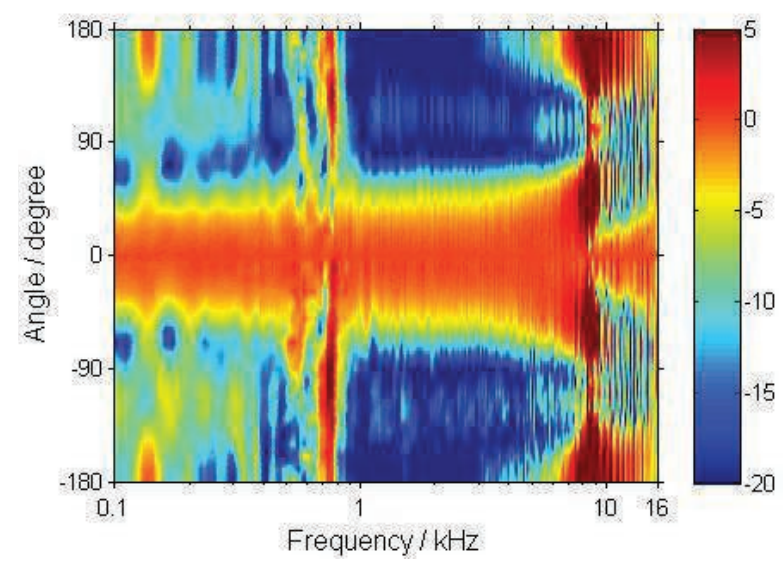

(d) Measured directivity map in $D=40 \mathrm{~cm}, d=27 \mathrm{~m}$

Fig. 8. Comparison of simulated and measured directivity maps for two different separations: $11 \mathrm{~mm}$ and $27 \mathrm{~mm}$. 


\section{Conclusions}

This paper has introduced the theoretical and experimental foundations of unidirectional sensor based on particle velocity gradient. A unidirectional sensor has been created by combining the signal of two closely spaced particle velocity components. Simulations were used to establish the relationship between frequency range, sensor spacing, sound source distance and measurement signal-to-noise ratio. Experimental results in a testing room have shown that the theoretical directivity can also be achieved in practice. Although the performance of the current measurement setup has limitations at low frequencies, the technology of unidirectional sensor based on particle velocity gradient has been proven feasible in a broadband range.

\section{References}

[1] $\mathrm{H}-\mathrm{E}$ de Bree, A particle velocity gradient beam forming system, The Journal of the Acoustical Society of America 127, 1820-1820 (2010); doi: 10.1121/1.3384208

[2] H.F. Olson, A uni-directional ribbon microphone, The Journal of the Acoustical Society of America 3, 315-316 (1932); doi: 10.1121/1.1901942

[3] H-E de Bree, P. Leussink, and T. Korthorst, H. Jansen, T. Lammerink, M. Elwenspoek, The $\mu$ flown: A novel device measuring acoustical flows, Solid-State Sensors and Actuators, 1995 and Eurosensors IX. Transducers '95. The 8th International Conference 1, 536-539 (1995); doi: 10.1109/SENSOR.1995.717278

[4] R.P. Glover, A review of cardioid type unidirectional microphones, The Journal of the Acoustical Society of America 11, 379 (1940) ; doi: 10.1121/1.1902162

[5] G.M. Sessler, J.E. West, Directional transducers, IEEE Transactions on Audio and Electroacoustics 19(1), 19-23 (1971); doi: 10.1109/TAU.1971.1162155 